\title{
THE EGG-SAC IN THE IDENTIFICATION OF SPECIES OF LATRODECTUS (BLACK-WIDOW SPIDERS) ${ }^{1}$
}

\author{
By J. W. Abalos \\ Instituto Nacional de Microbiología, \\ Santiago del Estero, Argentina
}

The genus Latrodectus comprises a few species very hard to classify. The geographical distribution of some spreads across several continents, making the problem even more complex and, at the same time, the synonymy richer. The difficulty lies in the lack of taxonomic characters which allow true differentiation among the species. Levi $\left(\right.$ I959) ${ }^{2}$ used for species identification, among other devices, the male genitalia as well as the female; thanks to this the problem of systematics of so ill-treated a group begins to be clearer.

In the course of the research we are carrying out on Latrodectus in Santiago del Estero (north-central region of Argentina) we have recognized the presence of five different species living in the same area. We were able to separate two of them according to their morphological characters; L. geometricus C. L. Koch, a domestic species we identified by its well-defined color and genitalic characters, among them the coils of the embolus of the male palpus, coils corresponding to those in the connecting ducts of the female. The other species, temporarily named Latrodectus No. I in order not to add further confusion of names, resembles $L$. curacaviensis (Müller) in the genitalic characters pointed out by Levi: the embolus of the male palpus and the connecting ducts of the female with two coils. The three remaining species correspond to what we call "L. mactans complex" with their three coiled embolus and female ducts. The morphological characters are not enough to separate these species. The width of the red spots on the abdomen guides the identification but is not reliable. The three of them live in the same area and ecologic environment. The only elements that allow us at present to separate these species in an accurate way are the size, shape and texture of the egg-sacs. We have temporarily called these species: Latrodectus No. 2, Latrodectus No. 3, and Latrodectus No. 4. However, many names are available for the species.

Latrodectus No. 2: The egg-sac is white when just formed, but usually turns yellowish in the course of days; it is spheroidal, slightly

\footnotetext{
${ }^{1}$ Research under the sponsorship of Consejo Nacional de Investigaciones Cientificas y Técnicas of Argentina.

${ }^{2}$ Levi, H. W. 1959. The spider genus Latrodectus (Araneae, Theridiidae)

Trans. Amer. Micros. Soc., $78(1): 7-43$.

Maniscript received by the editor October 31, 1962.
} 

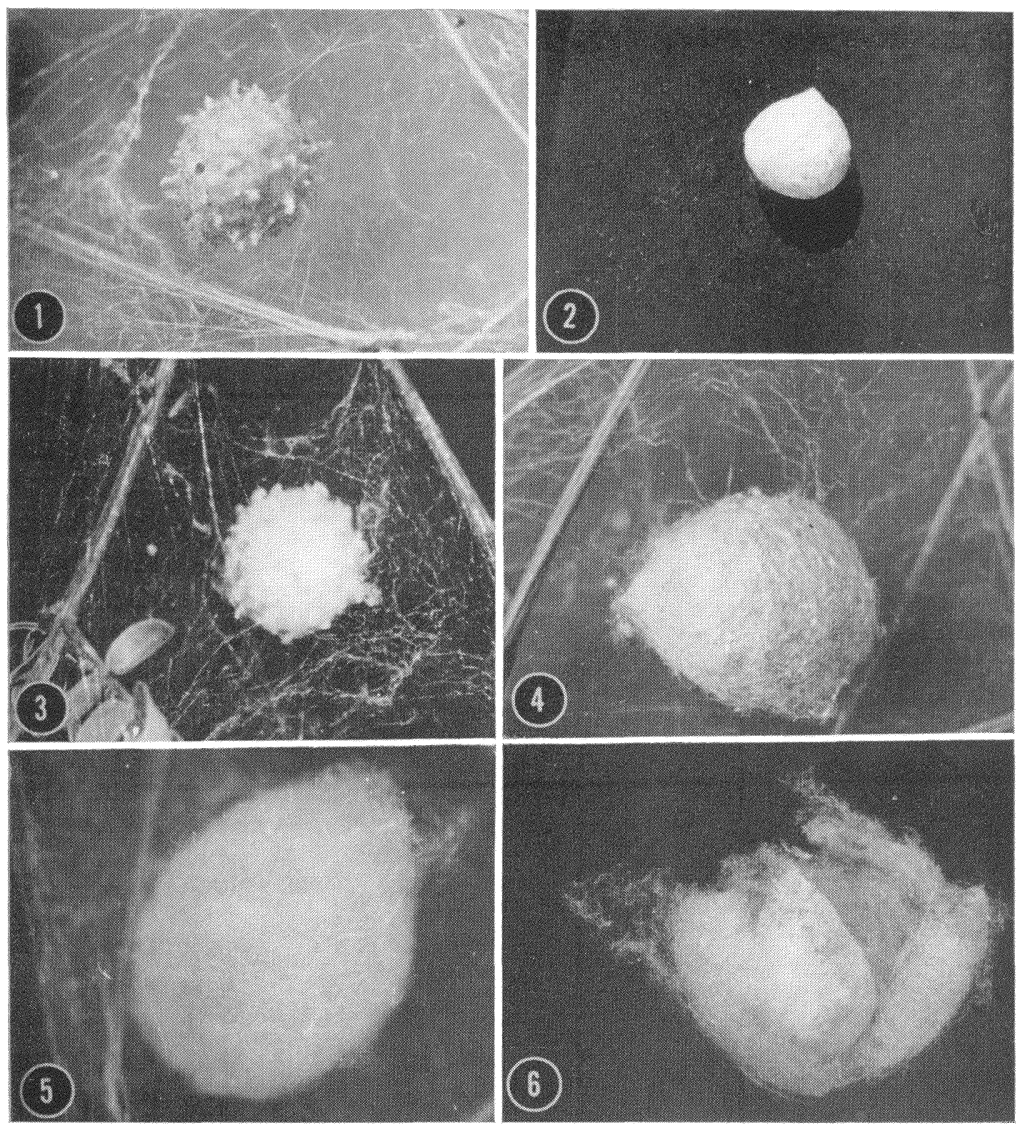

\section{Abalos - Latrodectus}

Figs. 1-6. Egg-sacs of Latrodectus. Fig. 1. Latrodectus geometricus C. L. Koch. Fig. 2. Latrodectus No. 1. Fig. 3. Latrodectus No. 2. Fig. 4. Latrodectus No. 3. Figs. 5-6. Latrodectus No. 4. 
pyriform and its most frequent size is about Io $\mathrm{mm}$. The sac is formed by threads 8 microns in diameter. They form a tissue, not very dense, but tight, that constitutes a thin cover, soft to pressure of the fingers. On the surface of the egg-sac we can observe numerous little web pompons that give a characteristic granulous aspect (Fig. 3). These little pompons are made by the spider with the hind legs; they are not firmly joined to the surface of the egg-sac and they usually fall off with handling.

Latrodectus No. 3: The egg-sac of this spider generally has the same shape as the one mentioned above, even though it sometimes changes, up to the point of presenting the aspect of an elongated pear; its size varies, but it is always bigger than the former species, reaching $2 \mathrm{Imm}$ in its biggest diameter. The sac wall is thicker and is formed by threads of a diameter of about 16 microns. These threads form a tight tissue, with a parchment texture that makes the egg-sac resistant to pressure. The egg-sac completely lacks pompons or any other ornament on its surface (Fig. 4).

Latrodectus No. 4: The egg-sac is white, pyriform, and its common size is about Io $\mathrm{mm}$. The sac-wall is thin, with threads of about 8 microns in diameter forming a less compact tissue. On this cover the spider weaves a coat about $3 \mathrm{~mm}$ thick, of loose tissue, that gives the egg-sac the appearance of a spherical woolen tuft (Figs. 5, 6).

We can also observe that Latrodectus No. I differs from the other species in its egg-sac. Its size is always smaller than in any of the former species, about $7 \mathrm{~mm}$, although it sometimes reaches Io $\mathrm{mm}$; its shape is spherical pyriform. Its color is white, often turning greyish in the course of days. The cover is thin with threads of about 8 microns in diameter, forming a very tight tissue of smooth surface, with a paper texture, and completely lacking exterior ornaments (Fig. 2). The egg-sac is fairly resistant to finger pressure.

The egg-sac of $\dot{L}$. geometricus, many times figured, is very characteristic: its color is whitish when just formed, turning yellowish in the course of days. It is spherical; its size about $10 \mathrm{~mm}$ in diameter; it is covered with conic protuberances that give it the curious aspect of a war-mace (Fig. I).

We are thankful to Dr. Herbert W. Levi, for his generosity and advice on the publication of this note and to Emilio Clementi for taking the photographs. 

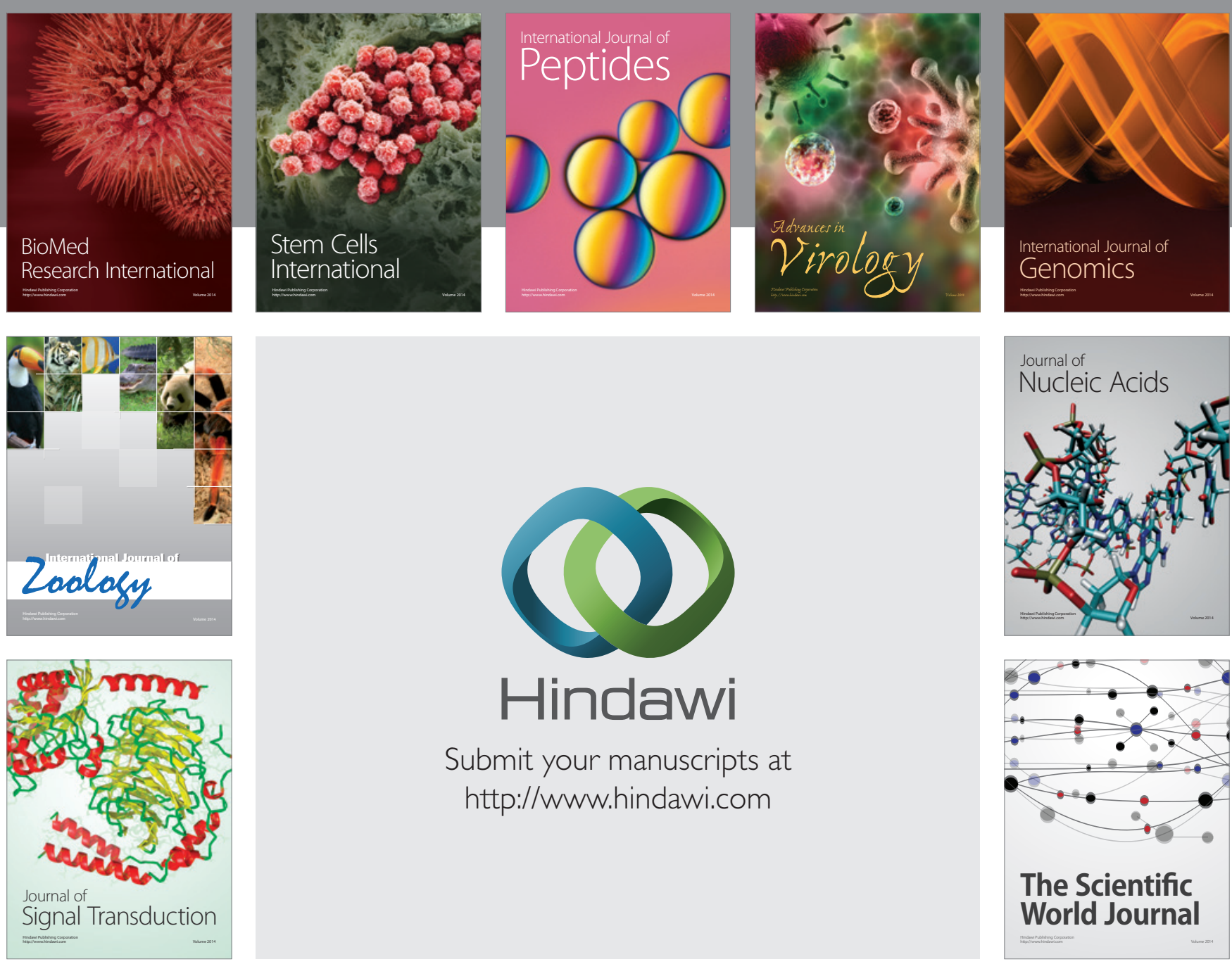

Submit your manuscripts at

http://www.hindawi.com
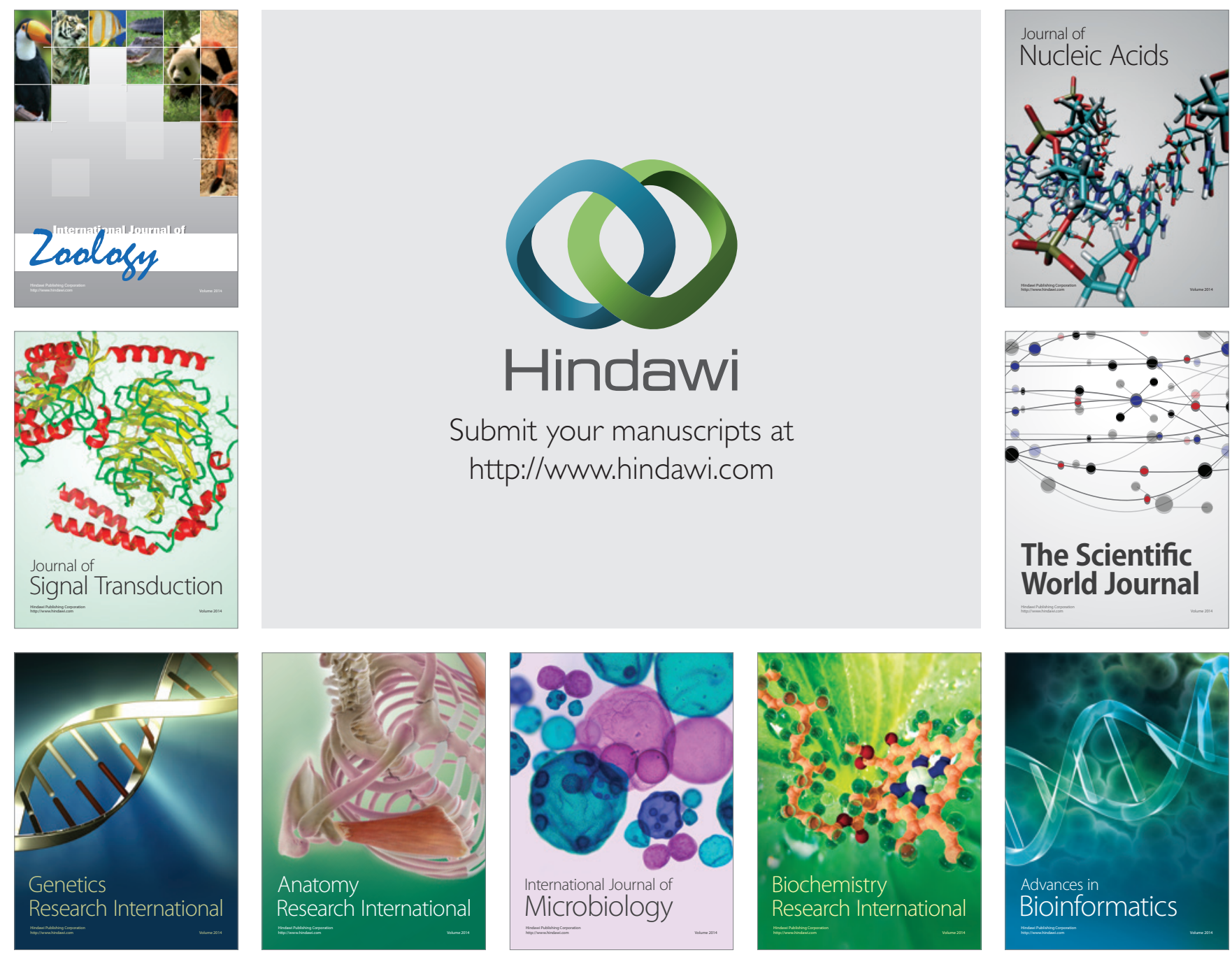

The Scientific World Journal
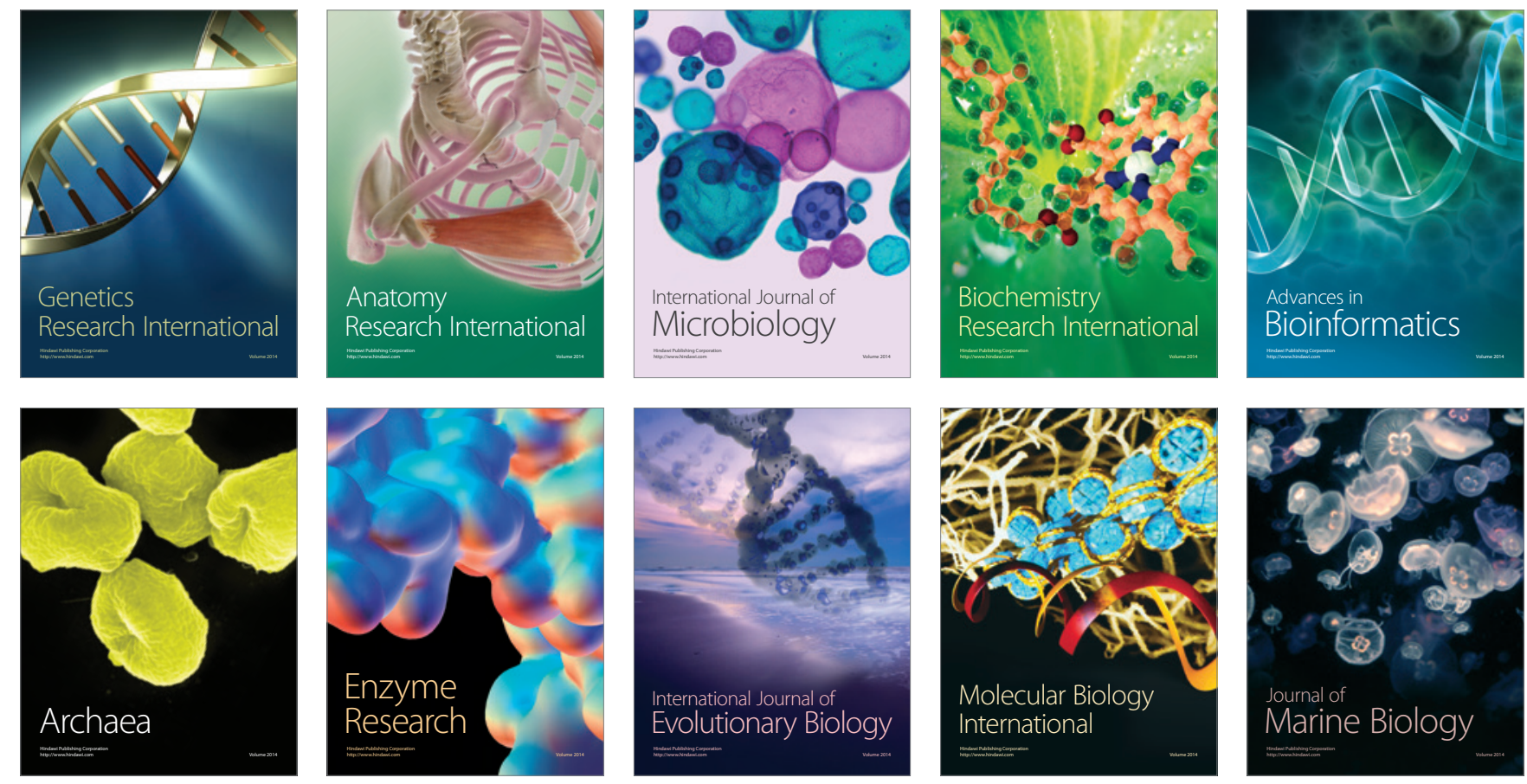